УДК 517.537 .72

O. M. Mulyava

\title{
ON THE RELATIVE GROWTH OF DIRICHLET SERIES WITH ZERO ABSCISSA OF ABSOLUTE CONVERGENCE
}

O. M. Mulyava. On the relative growth of Dirichlet series with zero abscissa of absolute convergence, Mat. Stud. 55 (2021), 44-50.

Let $F$ and $G$ be analytic functions given by Dirichlet series with exponents increasing to $+\infty$ and zero abscissa of absolute convergence. The growth of $F$ with respect to $G$ is studied in the generalized order

$$
\varrho_{\alpha, \beta}^{0}[F]_{G}=\varlimsup_{\sigma \uparrow 0} \frac{\alpha\left(1 / \mid M_{G}^{-1}\left(M_{F}(\sigma) \mid\right)\right.}{\beta(1 /|\sigma|)}
$$

and the generalized lower order

$$
\lambda_{\alpha, \beta}^{0}[F]_{G}=\varliminf_{\sigma \uparrow 0} \frac{\alpha\left(1 / \mid M_{G}^{-1}\left(M_{F}(\sigma) \mid\right)\right.}{\beta(1 /|\sigma|)},
$$

where $M_{F}(\sigma)=\sup \{|F(\sigma+i t)|: t \in \mathbb{R}\}, M_{G}^{-1}(x)$ is the function inverse to $M_{G}(\sigma)$ and $\alpha$ and $\beta$ are positive increasing to $+\infty$ functions. Formulas for computing these quantities are found.

Introduction. Let $f$ and $g$ be entire transcendental functions and $M_{f}(r)=\max \{|f(z)|$ : $|z|=r\}$. For the study of relative growth of the functions $f$ and $g$ Ch. Roy [1] used the order

$\varrho_{g}[f]=\varlimsup_{r \rightarrow+\infty} \frac{\ln M_{g}^{-1}\left(M_{f}(r)\right)}{\ln r}$ and the lower order $\lambda_{g}[f]=\varliminf_{r \rightarrow+\infty} \frac{\ln M_{g}^{-1}\left(M_{f}(r)\right)}{\ln r}$ of the function $f$ with respect to the function $g$. Research of relative growth of entire functions was continued by S.K. Data, T. Biswas and other mathematicians (see, for example, [2, 3, 4, 5]) in terms of maximal terms, Nevanlinna characteristic function and $k$-logarithmic orders. In [6] it is considered a relative growth of entire functions of two complex variables and in [7] the relative growth of entire Dirichlet series is studied in terms of $R$-orders.

Suppose that $\Lambda=\left(\lambda_{n}\right)$ is an increasing to $+\infty$ sequence of non-negative numbers and a Dirichlet series

$$
F(s)=\sum_{n=1}^{\infty} f_{n} \exp \left\{s \lambda_{n}\right\}, \quad s=\sigma+i t,
$$

has the abscissa of absolute convergence $\sigma_{a} \in(-\infty,+\infty]$. For $\sigma<\sigma_{a}$ we put $M_{F}(\sigma)=$ $\sup \{|F(\sigma+i t)|: t \in \mathbb{R}\}$. Suppose that $\sigma_{a}=+\infty$. Then the function $M_{F}(\sigma)$ is continuous and increasing to $+\infty$ on $(-\infty,+\infty)$ and, therefore, there exists the function $M_{F}^{-1}(x)$ inverse to $M_{F}(\sigma)$, which increases to $+\infty$ on $\left(x_{0},+\infty\right)$.

By $L$ we denote the class of continuous non-negative on $(-\infty,+\infty)$ functions $\alpha$ such that $\alpha(x)=\alpha\left(x_{0}\right) \geq 0$ for $x \leq x_{0}$ and $\alpha(x) \uparrow+\infty$ as $x_{0} \leq x \rightarrow+\infty$. We say that $\alpha \in L^{0}$,

2010 Mathematics Subject Classification: 30B50.

Keywords: Dirichlet series, relative growth, generalized order.

doi:10.30970/ms.55.1.44-50

(C) O. M. Mulyava, 2021 
if $\alpha \in L$ and $\alpha((1+o(1)) x)=(1+o(1)) \alpha(x)$ as $x \rightarrow+\infty$. Finally, $\alpha \in L_{s i}$, if $\alpha \in L$ and $\alpha(c x)=(1+o(1)) \alpha(x)$ as $x \rightarrow+\infty$ for each $c \in(0,+\infty)$, i. e. $\alpha$ is a slowly increasing function. Clearly, $L_{s i} \subset L^{0}$.

If $\alpha \in L, \beta \in L$ and $F$ is an entire function then the quantities

$$
\varrho_{\alpha, \beta}[F]:=\varlimsup_{\sigma \rightarrow+\infty} \frac{\alpha\left(\ln M_{F}(\sigma)\right)}{\beta(\sigma)}, \quad \lambda_{\alpha, \beta}[F]:=\varliminf_{\sigma \rightarrow+\infty} \frac{\alpha\left(\ln M_{F}(\sigma)\right)}{\beta(\sigma)}
$$

are called $([8,9])$ the generalized $(\alpha, \beta)$-order and the generalized lower $(\alpha, \beta)$-order of $F$, accordingly. We say that $F$ has the generalized regular $(\alpha, \beta)$-growth, if $0<\lambda_{\alpha, \beta}[F]=$ $=\varrho_{\alpha, \beta}[F]<+\infty$. The generalized $(\alpha, \beta)$-order $\varrho_{\alpha, \beta}[F]_{G}$ and the generalized lower $(\alpha, \beta)$ order $\lambda_{\alpha, \beta}[F]_{G}$ of the entire function $F$ with respect to an entire function $G$ given by Dirichlet series $G(s)=\sum_{n=1}^{\infty} g_{n} \exp \left\{s \lambda_{n}\right\}$ are defined [10] as follows

$$
\varrho_{\alpha, \beta}[F]_{G}:=\varlimsup_{\sigma \rightarrow+\infty} \frac{\alpha\left(M_{G}^{-1}\left(M_{F}(\sigma)\right)\right)}{\beta(\sigma)}, \lambda_{\alpha, \beta}[F]_{G}:=\varliminf_{\sigma \rightarrow+\infty} \frac{\alpha\left(M_{G}^{-1}\left(M_{F}(\sigma)\right)\right)}{\beta(\sigma)} .
$$

Connections between $\varrho_{\alpha, \beta}[F]_{G}$ and $\lambda_{\alpha, \beta}[F]_{G}$ on one hand and $\varrho_{\alpha, \beta}[F], \lambda_{\alpha, \beta}[F], \varrho_{\alpha, \beta}[G]$ and $\lambda_{\alpha, \beta}[F]$ on the other hand investigational in the articles $[10,11]$. In particular, in [10] the following elementary statements are proved: Let $\alpha \in L$ and $\beta \in L$.

$1^{0}$. Unless $\varrho_{\alpha, \beta}[F]=\varrho_{\alpha, \beta}[G]=0$ or $\varrho_{\alpha, \beta}[F]=\varrho_{\alpha, \beta}[G]=+\infty$, the inequality $\varrho_{\alpha, \beta}[F]_{G} \geq$ $\varrho_{\alpha, \beta}[F] / \varrho_{\alpha, \beta}[G]$ is true and subject to the condition of the the generalized regular $(\alpha, \beta)$ growth of $G$ this inequality converts into an equality.

$2^{0}$. Unless $\lambda_{\alpha, \beta}[F]=\lambda_{\alpha, \beta}[G]=0$ or $\lambda_{\alpha, \beta}[F]=\lambda_{\alpha, \beta}[G]=+\infty$, the inequality $\lambda_{\alpha, \beta}[F]_{G} \leq$ $\lambda_{\alpha, \beta}[F] / \lambda_{\alpha, \beta}[G]$ is true and subject to the condition of the the generalized regular $(\alpha, \beta)$ growth of $G$ this inequality converts into an equality.

The following theorem proved in paper [10] contains formulas for calculating the orders of relative growth in terms of the coefficients of the corresponding Dirichlet series (in terms of $R$-types the similar result is obtained in [11]).

Theorem 1 ([10]). Let $0<p<+\infty$ and one of the conditions is valid: a) $\ln n=o\left(\lambda_{n}\right)$ $(n \rightarrow \infty), \alpha \in L^{0}, \beta(\ln x) \in L^{0}, \frac{d \beta^{-1}(c \alpha(x))}{d \ln x} \rightarrow \frac{1}{p}(x \rightarrow+\infty)$ for each $\left.c \in(0,+\infty) ; \mathbf{b}\right) \alpha \in L_{s i}$, $\beta \in L^{0}, \varrho_{\alpha, \beta}[F]<+\infty, \frac{d \beta^{-1}(c \alpha(x))}{d \ln x}=O(1)(x \rightarrow+\infty)$ and $\ln n=o\left(\lambda_{n} \beta^{-1}\left(c \alpha\left(\lambda_{n}\right)\right)\right)(n \rightarrow \infty)$ for each $c \in(0,+\infty)$.

Suppose also that $\alpha\left(\lambda_{n+1} / p\right)=(1+o(1)) \alpha\left(\lambda_{n} / p\right)$ as $n \rightarrow \infty$. If the function $G$ has generalized regular $(\alpha, \beta)$-growth and $\varkappa_{n}[G]:=\frac{\ln \left|g_{n}\right|-\ln \left|g_{n+1}\right|}{\lambda_{n+1}-\lambda_{n}} \nearrow+\infty, n_{0} \leq n \rightarrow \infty$, then

$$
\varrho_{\alpha, \beta}[F]_{G}=\varlimsup_{n \rightarrow \infty} \beta\left(\frac{1}{p}+\frac{1}{\lambda_{n}} \ln \frac{1}{\left|g_{n}\right|}\right) / \beta\left(\frac{1}{p}+\frac{1}{\lambda_{n}} \ln \frac{1}{\left|f_{n}\right|}\right)
$$

unless $\varrho_{\alpha, \beta}[F]=\varrho_{\alpha, \beta}[G]=0$ or $\varrho_{\alpha, \beta}[F]=\varrho_{\alpha, \beta}[G]=+\infty$.

If, moreover, the function $G$ has generalized regular $(\alpha, \beta)$-growth and $\varkappa_{n}[F] \nearrow+\infty$ as $n_{0} \leq n \rightarrow \infty$ then

$$
\lambda_{\alpha, \beta}[F]_{G}=\varliminf_{n \rightarrow \infty} \beta\left(\frac{1}{p}+\frac{1}{\lambda_{n}} \ln \frac{1}{\left|g_{n}\right|}\right) / \beta\left(\frac{1}{p}+\frac{1}{\lambda_{n}} \ln \frac{1}{\left|f_{n}\right|}\right)
$$

unless $\lambda_{\alpha, \beta}[F]=\lambda_{\alpha, \beta}[G]=0$ or $\lambda_{\alpha, \beta}[F]=\lambda_{\alpha, \beta}[G]=+\infty$. 
Here we prove analogues of Theorem 1 for Dirichlet series with zero abscissa of absolute convergence.

1. Preliminary results. For $\alpha \in L$ and $\beta \in L$ the quantities

$$
\varrho_{\alpha, \beta}^{0}[F]=\varlimsup_{\sigma \uparrow 0} \frac{\alpha\left(\ln M_{F}(\sigma)\right)}{\beta(1 /|\sigma|)}, \quad \lambda_{\alpha, \beta}^{0}[F]=\varliminf_{\sigma \uparrow 0} \frac{\alpha\left(\ln M_{F}(\sigma)\right)}{\beta(1 /|\sigma|)} .
$$

are called ([12]) the generalized $(\alpha, \beta)$-order and the generalized lower $(\alpha, \beta)$-order of Dirichlet series (1) with zero abscissa of absolute convergence. As above, we say that $F$ has the generalized regular $(\alpha, \beta)$-growth, if $0<\lambda_{\alpha, \beta}^{0}[F]=\varrho_{\alpha, \beta}^{0}[F]<+\infty$. The function $M_{F}(\sigma)$ may be bounded on $(-\infty, 0)$, but if $\varlimsup_{n \rightarrow \infty}\left|f_{n}\right|=+\infty$ then $M_{F}(\sigma)$ is continuous and increasing to $+\infty$ on $(-\infty, 0)$ and, therefore, there exists the function $M_{F}^{-1}(x)$ inverse to $M_{F}(\sigma)$ which increases to 0 on $\left[x_{0},+\infty\right)$. In what follows, we will assume that the conditions $\varlimsup_{n \rightarrow \infty}\left|f_{n}\right|=$ $+\infty$ and $\varlimsup_{n \rightarrow \infty}\left|g_{n}\right|=+\infty$ are satisfied. By analogy to (3) we define the generalized $(\alpha, \beta)$ order and the generalized lower $(\alpha, \beta)$-order of $F$ with respect to $G$ as follows

$$
\varrho_{\alpha, \beta}^{0}[F]_{G}=\varlimsup_{\sigma \uparrow 0} \frac{\alpha\left(1 /\left|M_{G}^{-1}\left(M_{F}(\sigma)\right)\right|\right)}{\beta(1 /|\sigma|)}, \quad \lambda_{\alpha, \beta}^{0}[F]_{G}=\frac{\lim }{\sigma \uparrow 0} \frac{\alpha\left(1 /\left|M_{G}^{-1}\left(M_{F}(\sigma)\right)\right|\right)}{\beta(1 /|\sigma|)},
$$

and we say that $F$ has the generalized regular $(\alpha, \beta)$-growth with respect to $G$, if $0<$ $\lambda_{\alpha, \beta}^{0}[F]_{G}=\varrho_{\alpha, \beta}^{0}[F]_{G}<+\infty$.

We begin from the following proposition.

Proposition 1. If $\alpha \in L$ and $\beta \in L$ then: 1) the inequalities $\frac{\varrho_{\gamma, \beta}^{0}[F]}{\varrho_{\gamma, \alpha}^{0}[G]} \leq \varrho_{\alpha, \beta}^{0}[F]_{G} \leq \frac{\varrho_{\gamma, \beta}^{0}[F]}{\lambda_{\gamma, \alpha}^{0}[G]}$ are true for each function $\gamma \in L$ except for the cases $\varrho_{\gamma, \beta}^{0}[F]=\varrho_{\gamma, \alpha}^{0}[G]=0, \varrho_{\gamma, \beta}^{0}[F]=$ $=\lambda_{\gamma, \alpha}^{0}[G]=0, \varrho_{\gamma, \beta}^{0}[F]=\varrho_{\gamma, \alpha}^{0}[G]=+\infty, \varrho_{\gamma, \beta}^{0}[F]=\lambda_{\gamma, \alpha}^{0}[G]=+\infty$;

2) the inequalities $\frac{\lambda_{\gamma, \beta}^{0}[F]}{\varrho_{\gamma, \alpha}^{0}[G]} \leq \lambda_{\alpha, \beta}^{0}[F]_{G} \leq \frac{\lambda_{\gamma, \beta}^{0}[F]}{\lambda_{\gamma, \alpha}^{0}[G]} \quad$ are true for each function $\gamma \in L$ except for the cases $\lambda_{\gamma, \beta}^{0}[F]=\lambda_{\gamma, \alpha}^{0}[G]=0, \lambda_{\gamma, \beta}^{0}[F]=\varrho_{\gamma, \alpha}^{0}[G]=0, \lambda_{\gamma, \beta}^{0}[F]=\lambda_{\gamma, \alpha}^{0}[G]=+\infty$, $\lambda_{\gamma, \beta}^{0}[F]=\varrho_{\gamma, \alpha}^{0}[G]=+\infty$.

Proof. Using the inequalities $\varlimsup \lim a \cdot \underline{\lim } b \leq \varlimsup \lim a b \leq \varlimsup \lim a \cdot \varlimsup \lim b$, by definition of $\varrho_{\alpha, \beta}^{0}[F]_{G}$, $\varrho_{\gamma, \beta}^{0}[F], \varrho_{\gamma, \alpha}^{0}[G]$ and $\lambda_{\gamma, \alpha}^{0}[G]$, we obtain

and

$$
\begin{gathered}
\varrho_{\alpha, \beta}^{0}[F]_{G}=\varlimsup_{x \rightarrow+\infty} \frac{\alpha\left(1 /\left|M_{G}^{-1}(x)\right|\right)}{\beta\left(1 /\left|M_{F}^{-1}(x)\right|\right)}=\varlimsup_{x \rightarrow+\infty} \frac{\gamma(\ln x)}{\beta\left(1 /\left|M_{F}^{-1}(x)\right|\right)} \frac{\alpha\left(1 /\left|M_{G}^{-1}(x)\right|\right)}{\gamma(\ln x)} \geq \\
\geq \varlimsup_{x \rightarrow+\infty} \frac{\gamma(\ln x)}{\beta\left(1 /\left|M_{F}^{-1}(x)\right|\right)} \underset{x \rightarrow+\infty}{\varliminf_{G}} \frac{\alpha\left(1 /\left|M_{G}^{-1}(x)\right|\right)}{\gamma(\ln x)}=\varrho_{\gamma, \beta}[F] / \varrho_{\gamma, \alpha}[G]
\end{gathered}
$$

$$
\varrho_{\alpha, \beta}^{0}[F]_{G} \leq \varlimsup_{x \rightarrow+\infty} \frac{\gamma(\ln x)}{\beta\left(1 /\left|M_{F}^{-1}(x)\right|\right)} \varlimsup_{x \rightarrow+\infty} \frac{\alpha\left(1 /\left|M_{G}^{-1}(x)\right|\right)}{\gamma(\ln x)}=\varrho_{\gamma, \beta}^{0}[F] / \lambda_{\gamma, \alpha}^{0}[G],
$$

i.e. the inequalities from the statement 1) are proved.

The proof of inequalities from the statement 2) is completely analogous to the previous one and follows from the inequalities $\underline{\lim } a \cdot \underline{\lim } b \leq \underline{\lim } a b \leq \overline{\lim } a \cdot \underline{\lim } b$, by the definitions of $\lambda_{\gamma, \beta}^{0}[F], \varrho_{\gamma, \alpha}^{0}[G], \lambda_{\alpha, \beta}^{0}[F]_{G}, \lambda_{\gamma, \alpha}^{0}[G]$.

Remark 1. In the statements 1) and 2) of Proposition 1 the conditions for the function $\gamma$ hold if $0<\lambda_{\gamma, \alpha}^{0}[G] \leq \varrho_{\gamma, \alpha}^{0}[G]<+\infty$. From Proposition 1 it follows that if $G$ has the generalized regular $(\gamma, \alpha)$-growth then $\varrho_{\alpha, \beta}^{0}[F]_{G}=\varrho_{\gamma, \beta}^{0}[F] / \varrho_{\gamma, \alpha}^{0}[G]$ and $\lambda_{\alpha, \beta}^{0}[F]_{G}=\lambda_{\gamma, \beta}^{0}[F] / \lambda_{\gamma, \alpha}^{0}[G]$. 
The most commonly used growth characteristics of Dirichlet series with zero abscissa of absolute convergence are the order $\varrho^{0}[F]=\varlimsup_{\sigma \uparrow 0} \frac{\left.\ln \ln M_{F}(\sigma)\right)}{\ln (1 /|\sigma|)}$, the lower order $\lambda^{0}[F]=\varliminf_{\sigma \uparrow 0} \frac{\left.\ln \ln M_{F}(\sigma)\right)}{\ln (1 /|\sigma|)}$, the type $T^{0}[F]=\varlimsup_{\sigma \uparrow 0}|\sigma|^{\varrho^{0}[F]} \ln M_{F}(\sigma)$ and the lower type $t^{0}[F]=\varliminf_{\sigma \uparrow 0}|\sigma|^{\varrho^{0}[F]} \ln M_{F}(\sigma)$, provided $0<\varrho^{0}[F]<+\infty$.

Put $\alpha(x)=\beta(x)=\gamma(x)=\ln ^{+} x$. Then from Proposition 1 we obtain the following statement by simple calculations.

Corollary 1. If $0<\lambda^{0}[G] \leq \varrho^{0}[G]<+\infty$ then

$$
\begin{aligned}
& \varrho^{0}[F] / \varrho^{0}[G] \leq \varrho^{0}[F]_{G}:=\varlimsup_{\sigma \uparrow 0} \frac{\ln \left(1 / \mid M_{G}^{-1}\left(M_{F}(\sigma) \mid\right)\right.}{\ln (1 /|\sigma|)} \leq \varrho^{0}[F] / \lambda^{0}[G], \\
& \lambda^{0}[F] / \varrho^{0}[G] \leq \lambda^{0}[F]_{G}:=\varlimsup_{\sigma \uparrow 0} \frac{\ln \left(1 /\left|M_{G}^{-1}\left(M_{F}(\sigma)\right)\right|\right.}{\ln (1 /|\sigma|)} \leq \lambda^{0}[F] / \lambda^{0}[G] .
\end{aligned}
$$

If $0<\varrho^{0}[F]_{G}<+\infty$ then we define the type and the lower type of the function $F$ with respect to the function $G$ as follows

$$
T^{0}[F]_{G}=\varlimsup_{\sigma \uparrow 0} \frac{|\sigma|^{\varrho^{0}[F]_{G}}}{\mid M_{G}^{-1}\left(M_{F}(\sigma) \mid\right.}, \quad t^{0}[F]_{G}=\underline{\lim _{\sigma \uparrow 0}} \frac{|\sigma|^{\varrho^{0}[F]_{G}}}{\mid M_{G}^{-1}\left(M_{F}(\sigma) \mid\right.} .
$$

Proposition 2. If the function $G$ has the regular growth (i. e. $0<\lambda^{0}[G]=\varrho^{0}[G]<+\infty$ ) and $0<t^{0}[G] \leq T^{0}[G]<+\infty$ then

$$
T^{0}[F] / T^{0}[G] \leq\left(T^{0}[F]_{G}\right)^{\varrho^{0}[G]} \leq T^{0}[F] / t^{0}[G], \quad t^{0}[F] / T^{0}[G] \leq\left(t^{0}[F]_{G}\right)^{\varrho^{0}[G]} \leq t^{0}[F] / t^{0}[G] .
$$

Proof. Since $G$ has the regular growth, by Proposition $1 \varrho^{0}[F]_{G}=\varrho^{0}[F] / \varrho^{0}[G]$. Therefore,

$$
\begin{gathered}
\left(T^{0}[F]_{G}\right)^{\varrho^{0}[G]}=\left(\varlimsup_{x \rightarrow+\infty} \frac{\left|M_{F}^{-1}(x)\right|^{\varrho^{0}[F] / \varrho^{0}[G]}}{\left|M_{G}^{-1}(x)\right|}\right)^{\varrho^{0}[G]}=\varlimsup_{x \rightarrow+\infty} \frac{\left|M_{F}^{-1}(x)\right|^{\varrho^{0}[F]}}{\left|M_{G}^{-1}(x)\right|^{/ \varrho^{0}[G]}}= \\
=\varlimsup_{\sigma \uparrow 0}\left(|\sigma|^{\varrho^{0}[F]} \ln M_{F}(\sigma)\right) \varlimsup_{x \rightarrow+\infty} \frac{1}{|\sigma|^{\varrho^{0}[G]} \ln M_{G}(\sigma)}=T^{0}[F] / t^{0}[G], \\
\left(T^{0}[F]_{G}\right)^{\varrho^{0}[G]} \geq \varlimsup_{x \rightarrow+\infty}\left(\left|M_{F}^{-1}(x)\right|^{\varrho^{0}[F]} \ln x\right) \underset{x \rightarrow+\infty}{\lim _{G} \frac{1}{\left.\left|M_{G}^{-1}(x)\right|\right|^{0}[G]} \ln x}=T^{0}[F] / T^{0}[G] .
\end{gathered}
$$

This completes the proof of the first two inequalities. The other two inequalities are proved similarly simply.

2. The main result. In order to obtain analogues of Theorem 1, we need formulas for finding generalized orders in terms of the coefficients of the Dirichlet series. The following lemma holds [13].

Lemma 1. Let Dirichlet series (1) have the abscissa of absolute convergence $\sigma_{a}=0$. Suppose that $\gamma \in L_{s i}, \beta \in L_{s i}$ and for each $c \in(0,+\infty)$

$$
\frac{x}{\beta^{-1}(c \gamma(x))} \uparrow+\infty, \quad \gamma\left(\frac{x}{\beta^{-1}(c \gamma(x))}\right)=(1+o(1)) \gamma(x), \quad x \rightarrow+\infty .
$$

If $\gamma\left(\lambda_{n}\right)=o\left(\beta\left(\frac{\lambda_{n}}{\ln n}\right)\right)$ as $n \rightarrow \infty$ then $\varrho_{\gamma, \beta}^{0}[F]=\varlimsup_{n \rightarrow \infty} \frac{\gamma\left(\lambda_{n}\right)}{\beta\left(\lambda_{n} / \ln \left|f_{n}\right|\right)}$. If, moreover, $\gamma\left(\lambda_{n+1}\right) \sim \gamma\left(\lambda_{n}\right)$ and $\varkappa_{n}[F] \nearrow 0$ as $n_{0} \leq n \rightarrow \infty$ then $\lambda_{\gamma, \beta}^{0}[F]=\varliminf_{n \rightarrow \infty} \frac{\gamma\left(\lambda_{n}\right)}{\beta\left(\lambda_{n} / \ln \left|f_{n}\right|\right)}$. 
From conditions (5) it follows that the function $\gamma$ grows more slowly than the function $\beta$. In the case when the function $\beta$ grows more slowly than the function $\gamma$, the following lemma is true $([13])$.

Lemma 2. Let Dirichlet series (1) have the abscissa of absolute convergence $\sigma_{a}=0$. Suppose that $\gamma \in L_{s i}, \beta \in L_{s i}$ and for each $c \in(0,+\infty)$

$$
\frac{x}{\gamma^{-1}(c \beta(x))} \uparrow+\infty, \quad \gamma\left(\frac{x}{\gamma^{-1}(c \beta(x))}\right)=(1+o(1)) \beta(x), \quad x \rightarrow+\infty .
$$

If $\gamma(\ln n)=o\left(\beta\left(\lambda_{n}\right)\right)$ as $n \rightarrow \infty$ then $\varrho_{\gamma, \beta}^{0}[F]=\varlimsup_{n \rightarrow \infty} \frac{\gamma\left(\ln \left|f_{n}\right|\right)}{\beta\left(\lambda_{n}\right)}$. If, moreover, $\beta\left(\lambda_{n+1}\right) \sim \beta\left(\lambda_{n}\right)$ and $\varkappa_{n}[F] \nearrow 0$ as $n_{0} \leq n \rightarrow \infty$ then $\lambda_{\gamma, \beta}^{0}[F]=\varliminf_{n \rightarrow \infty} \frac{\gamma\left(\ln \left|f_{n}\right|\right)}{\beta\left(\lambda_{n}\right)}$.

Using Lemma 1 and Proposition 1 we prove the following theorem.

Theorem 2. Let the functions $\gamma \in L_{s i}, \beta \in L_{s i}$ and $\alpha \in L_{s i}$ satisfy conditions (5) and the same conditions with replacement of $\beta$ by $\alpha$. Suppose that $\gamma\left(\lambda_{n}\right)=o\left(\beta\left(\lambda_{n} / \ln n\right)\right)$ and $\gamma\left(\lambda_{n}\right)=o\left(\alpha\left(\lambda_{n} / \ln n\right)\right)$ as $n \rightarrow \infty$. If the function $G$ has the generalized regular $(\gamma, \alpha)$ growth, $\gamma\left(\lambda_{n+1}\right) \sim \gamma\left(\lambda_{n}\right)$ and $\varkappa_{n}[G] \nearrow 0$ as $n_{0} \leq n \rightarrow \infty$ then

$$
\varrho_{\alpha, \beta}^{0}[F]_{G}=P_{\alpha, \beta}:=\varlimsup_{n \rightarrow \infty} \frac{\alpha\left(\lambda_{n} / \ln \left|g_{n}\right|\right)}{\beta\left(\lambda_{n} / \ln \left|f_{n}\right|\right)} \text {. }
$$

If, moreover, $\varkappa_{n}[F] \nearrow 0$ as $n_{0} \leq n \rightarrow \infty$ then

$$
\lambda_{\alpha, \beta}^{0}[F]_{G}=p_{\alpha, \beta}:=\varliminf_{n \rightarrow \infty} \frac{\alpha\left(\lambda_{n} / \ln \left|g_{n}\right|\right)}{\beta\left(\lambda_{n} / \ln \left|f_{n}\right|\right)} .
$$

Proof. Since the function $G$ has the generalized regular $(\gamma, \alpha)$-growth, by Proposition 1 and Lemma 1 we have

$$
\varrho_{\alpha, \beta}^{0}[F]_{G}=\frac{\varrho_{\gamma, \beta}^{0}[F]}{\varrho_{\gamma, \alpha}^{0}[G]}=\varlimsup_{n \rightarrow \infty} \frac{\gamma\left(\lambda_{n}\right)}{\beta\left(\lambda_{n} / \ln \left|f_{n}\right|\right)} \varliminf_{n \rightarrow \infty} \frac{\alpha\left(\lambda_{n} / \ln \left|g_{n}\right|\right)}{\gamma\left(\lambda_{n}\right)} \leq \varlimsup_{n \rightarrow \infty} \frac{\alpha\left(\lambda_{n} / \ln \left|g_{n}\right|\right)}{\beta\left(\lambda_{n} / \ln \left|f_{n}\right|\right)}=P_{\alpha, \beta} .
$$

On the other hand, let $P_{\alpha, \beta}>0$. Then for every $\varepsilon \in\left(0, P_{\alpha, \beta}\right)$ there exists an increasing to $+\infty$ sequence $\left(n_{k}\right)$ of integers such that $\alpha\left(\lambda_{n_{k}} / \ln \left|g_{n_{k}}\right|\right) \geq\left(P_{\alpha, \beta}-\varepsilon\right) \beta\left(\lambda_{n_{k}} / \ln \left|f_{n_{k}}\right|\right)$, i. e.

$$
\frac{\gamma\left(\lambda_{n_{k}}\right)}{\beta\left(\lambda_{n_{k}} / \ln \left|f_{n_{k}}\right|\right)} \geq\left(P_{\alpha, \beta}-\varepsilon\right) \frac{\gamma\left(\lambda_{n_{k}}\right)}{\alpha\left(\lambda_{n_{k}} / \ln \left|g_{n_{k}}\right|\right)}
$$

and, thus, by Lemma 1 and Proposition 1

$$
\begin{aligned}
\varrho_{\gamma, \beta}^{0}[F] & =\varlimsup_{n \rightarrow \infty} \frac{\gamma\left(\lambda_{n}\right)}{\beta\left(\lambda_{n} / \ln \left|f_{n}\right|\right)} \geq \varlimsup_{k \rightarrow \infty} \frac{\gamma\left(\lambda_{n_{k}}\right)}{\beta\left(\lambda_{n_{k}} / \ln \left|f_{n_{k}}\right|\right)} \geq\left(P_{\alpha, \beta}-\varepsilon\right) \varlimsup_{k \rightarrow \infty} \frac{\gamma\left(\lambda_{n_{k}}\right)}{\alpha\left(\lambda_{n_{k}} / \ln \left|g_{n_{k}}\right|\right)} \geq \\
& \geq\left(P_{\alpha, \beta}-\varepsilon\right) \underline{\lim _{n \rightarrow \infty}} \frac{\gamma\left(\lambda_{n}\right)}{\alpha\left(\lambda_{n} / \ln \left|g_{n}\right|\right)}=\left(P_{\alpha, \beta}-\varepsilon\right) \lambda_{\gamma, \alpha}^{0}[G]=\left(P_{\alpha, \beta}-\varepsilon\right) \varrho_{\gamma, \alpha}^{0}[G],
\end{aligned}
$$

whence in view of the arbitrariness of $\varepsilon$ we get $\varrho_{\alpha, \beta}^{0}[F]_{G} \geq P_{\alpha, \beta}$. For $P_{\alpha, \beta}=0$ the last inequality is obvious. The first statement of Theorem 2 is proved. 
For the proof of the second statement of Theorem 2, we remark that since $G$ has generalized regular $(\gamma, \alpha)$-growth, by Theorem 1 and Lemma 1

$$
\lambda_{\alpha, \beta}^{0}[F]_{G}=\frac{\lambda_{\gamma, \beta}^{0}[F]}{\lambda_{\gamma, \alpha}^{0}[G]}=\varliminf_{n \rightarrow \infty} \frac{\gamma\left(\lambda_{n}\right)}{\beta\left(\lambda_{n} / \ln \left|f_{n}\right|\right)} \varlimsup_{n \rightarrow \infty} \frac{\alpha\left(\lambda_{n} / \ln \left|g_{n}\right|\right)}{\gamma\left(\lambda_{n}\right)} \geq \underset{n \rightarrow \infty}{\lim _{n \rightarrow \infty}} \frac{\alpha\left(\lambda_{n} / \ln \left|g_{n}\right|\right)}{\beta\left(\lambda_{n} / \ln \left|f_{n}\right|\right)}=p_{\alpha, \beta} .
$$

On the other hand, let $p_{\alpha, \beta}<+\infty$. Then for every $\varepsilon>0$ there exists an increasing to $+\infty$ sequence $\left(n_{k}\right)$ of integers such that $\alpha\left(\lambda_{n_{k}} / \ln \left|g_{n_{k}}\right|\right) \leq\left(p_{\alpha, \beta}+\varepsilon\right) \beta\left(\lambda_{n_{k}} / \ln \left|f_{n_{k}}\right|\right)$ and, as above,

$$
\begin{gathered}
\lambda_{\gamma, \beta}^{0}[F] \leq \lim _{k \rightarrow \infty} \frac{\gamma\left(\lambda_{n_{k}}\right)}{\beta\left(\lambda_{n_{k}} / \ln \left|f_{n_{k}}\right|\right)} \leq\left(p_{\alpha, \beta}+\varepsilon\right) \underline{\lim _{k \rightarrow \infty}} \frac{\gamma\left(\lambda_{n_{k}}\right)}{\alpha\left(\lambda_{n_{k}} / \ln \left|g_{n_{k}}\right|\right)} \leq \\
\leq\left(p_{\alpha, \beta}+\varepsilon\right) \varlimsup_{n \rightarrow \infty} \frac{\gamma\left(\lambda_{n}\right)}{\alpha\left(\lambda_{n} / \ln \left|g_{n}\right|\right)}=\left(p_{\alpha, \beta}+\varepsilon\right) \varrho_{\gamma, \alpha}^{0}[G]=\left(p_{\alpha, \beta}+\varepsilon\right) \lambda_{\gamma, \alpha}^{0}[G],
\end{gathered}
$$

whence in view of the arbitrariness of $\varepsilon$ we get $\lambda_{\alpha, \beta}^{0}[F]_{G} \leq p_{\alpha, \beta}$. For $p_{\alpha, \beta}=+\infty$ the last inequality is obvious.

Using Lemma 2 and Proposition 1 now we prove the following theorem.

Theorem 3. Let the functions $\gamma \in L_{s i}, \beta \in L_{s i}$ and $\alpha \in L_{s i}$ satisfy conditions (6) and the same conditions with replacement of $\beta$ by $\alpha$. Suppose that $\gamma(\ln n)=o\left(\beta\left(\lambda_{n}\right)\right)$ and $\gamma(\ln n)=o\left(\alpha\left(\lambda_{n}\right)\right)$ as $n \rightarrow \infty$. If the function $G$ has the generalized regular $(\gamma, \alpha)$-growth, $\alpha\left(\lambda_{n+1}\right) \sim \alpha\left(\lambda_{n}\right)$ and $\varkappa_{n}[G] \nearrow 0$ as $n_{0} \leq n \rightarrow \infty$ then

$$
\varrho_{\alpha, \beta}^{0}[F]_{G}=Q_{\alpha, \beta}:=\varlimsup_{n \rightarrow \infty} \frac{\alpha\left(\lambda_{n}\right) \gamma\left(\ln \left|f_{n}\right|\right)}{\beta\left(\lambda_{n}\right) \gamma\left(\ln \left|g_{n}\right|\right)} .
$$

If, moreover, $\beta\left(\lambda_{n+1}\right) \sim \beta\left(\lambda_{n}\right)$ and $\varkappa_{n}[F] \nearrow 0$ as $n_{0} \leq n \rightarrow \infty$ then

$$
\lambda_{\alpha, \beta}^{0}[F]_{G}=q_{\alpha, \beta}:=\varliminf_{n \rightarrow \infty} \frac{\alpha\left(\lambda_{n}\right) \gamma\left(\ln \left|f_{n}\right|\right)}{\beta\left(\lambda_{n}\right) \gamma\left(\ln \left|g_{n}\right|\right)} .
$$

Proof. Since the function $G$ has the generalized regular $(\gamma, \alpha)$-growth, by Proposition 1 and Lemma 1 we have

$$
\varrho_{\alpha, \beta}^{0}[F]_{G}=\frac{\varrho_{\gamma, \beta}^{0}[F]}{\varrho_{\gamma, \alpha}^{0}[G]}=\varlimsup_{n \rightarrow \infty} \frac{\gamma\left(\ln \left|f_{n}\right|\right)}{\beta\left(\lambda_{n}\right)} \varliminf_{n \rightarrow \infty} \frac{\alpha\left(\lambda_{n}\right)}{\gamma\left(\ln \left|g_{n}\right|\right)} \leq \varlimsup_{n \rightarrow \infty} \frac{\alpha\left(\lambda_{n}\right) \gamma\left(\ln \left|f_{n}\right|\right)}{\beta\left(\lambda_{n}\right) \gamma\left(\ln \left|g_{n}\right|\right)}=Q_{\alpha, \beta} .
$$

On the other hand, if $Q_{\alpha, \beta}>0$ then for every $\varepsilon \in\left(0, Q_{\alpha, \beta}\right)$ there exists an increasing to $+\infty$ sequence $\left(n_{k}\right)$ of integers such that $\frac{\gamma\left(\ln \left|f_{n_{k}}\right|\right)}{\beta\left(\lambda_{n_{k}}\right)} \geq\left(Q_{\alpha, \beta}-\varepsilon\right) \frac{\gamma\left(\ln \left|g_{n_{k}}\right|\right)}{\alpha\left(\lambda_{n_{k}}\right)}$, whence by Lemma 2 and Proposition 1

$$
\begin{gathered}
\varrho_{\gamma, \beta}^{0}[F] \geq \varlimsup_{k \rightarrow \infty} \frac{\gamma\left(\ln \left|f_{n_{k}}\right|\right)}{\beta\left(\lambda_{n_{k}}\right)} \geq\left(Q_{\alpha, \beta}-\varepsilon\right) \varlimsup_{k \rightarrow \infty} \frac{\gamma\left(\ln \left|g_{n_{k}}\right|\right)}{\alpha\left(\lambda_{n_{k}}\right)} \geq \\
\geq\left(Q_{\alpha, \beta}-\varepsilon\right) \varliminf_{k \rightarrow \infty} \frac{\gamma\left(\ln \left|g_{n}\right|\right)}{\alpha\left(\lambda_{n}\right)}=\left(Q_{\alpha, \beta}-\varepsilon\right) \lambda_{\gamma, \alpha}^{0}[G]=\left(Q_{\alpha, \beta}-\varepsilon\right) \varrho_{\gamma, \alpha}^{0}[G],
\end{gathered}
$$

and, thus, in view of the arbitrariness of $\varepsilon$ we get $\varrho_{\alpha, \beta}^{0}[F]_{G} \geq Q_{\alpha, \beta}$. For $Q_{\alpha, \beta}=0$ the last inequality is obvious. The first equality is proved.

The second equality is also easy to prove.

The functions $\alpha(x)=\beta(x)=\gamma(x)=\ln ^{+} x$ do not satisfy the conditions of any of Theorems 2 and 3. But to study the relative growth in terms of order and lower order, we can use the following lemma [14]. 
Lemma 3. Let Dirichlet series (1) has the abscissa of absolute convergence $\sigma_{a}=0$. If $\left.\ln \ln n=o\left(\ln \lambda_{n}\right)\right)$ as $n \rightarrow \infty$ then $\varrho^{0}[F]=\frac{\alpha^{*}[f]}{1-\alpha^{*}[f]}$, where $\alpha^{*}[f]=\varlimsup_{n \rightarrow \infty} \frac{\ln ^{+} \ln \left|f_{n}\right|}{\ln \lambda_{n}}$. If, moreover, $\ln \lambda_{n+1} \sim \ln \lambda_{n}$ and $\varkappa_{n}[F] \nearrow 0$ as $n_{0} \leq n \rightarrow \infty$ then $\lambda^{0}[F]=\frac{\alpha_{*}}{1-\alpha_{*}}$, where $\alpha_{*}[f]=$ $\varliminf_{n \rightarrow \infty} \frac{\ln ^{+} \ln \left|f_{n}\right|}{\ln \lambda_{n}}$.

If the function $G$ has the regular growth then by Corollary $1 \varrho^{0}[F]_{G}=\varrho^{0}[F]$ and $\lambda^{0}[F]_{G}=$ $\lambda^{0}[F]$. Therefore, Lemma 3 implies the following statement.

Proposition 3. If the function $G$ has the regular growth and $\left.\ln \ln n=o\left(\ln \lambda_{n}\right)\right)$ as $n \rightarrow \infty$ then $\varrho^{0}[F]_{G}=\alpha^{*} /\left(1-\alpha^{*}\right)$. If, moreover, $\ln \lambda_{n+1} \sim \ln \lambda_{n}$ and $\varkappa_{n}[F] \nearrow 0$ as $n_{0} \leq n \rightarrow \infty$ then $\lambda^{0}[F]_{G}=\alpha_{*} /\left(1-\alpha_{*}\right)$.

\section{REFERENCES}

1. Ch. Roy, On the relative order and lower order of an entire functiion// Bull. Soc. Cal. Math. Soc. 2010. - V.102, №1 - P. 17-26.

2. S.K. Data, A.R. Maji, Relative order of entire functions in terms of their maximum terms// Int. Journal of Math. Analysis. - 2011. - V.5, №43. - P. 2119-2126.

3. S.K. Data, T. Biswas, Ch. Ghosh, Growth analysis of entire functions concerning generalized relative type and generalized relative weak type// Facta Univ.(NIS). Ser. Math. Inform. - 2015. - V.30, №3.- P. $295-324$.

4. S.K. Data, T. Biswas, A. Hoque, Some results on the growth analysis of entire function using their maximum terms and relative $L^{*}$-order// Journ. Math. Extension. - 2016. - V.10, №2. - P. 59-73.

5. S.K. Data, T. Biswas, P. Das, Some results on generalized relative order of meromorohic functions// Ufa Math. Journ. - 2016. - V.8, №2. - P. 92-103.

6. S.K. Data, T. Biswas, Growth analysis of entire functions of two complex variables// Sahand Communications in Math. Analysis. - 2016. - V.3, №2. - P. 13-22.

7. S.K. Data, T. Biswas, Some growth analysis of entire functions in the form of vector valued Dirichlet series on the basis on their relative Ritt $L^{*}$-order and relative Ritt $L^{*}$-lower order// New Trends in Math. Sci. 2017. - V.5, №2. - P. 97-103.

8. Ja.D. Pyanylo, M.M. Sheremeta, On the growth of entire fuctions given by Dirichlet series// Izv. vuzov, Mat. - 1975. - V.10. - P. 91-93. (in Russian)

9. M.M. Sheremeta, Entire Dirichlet series. - Kyiv: ISDO. - 1993. - 168 p. (in Ukrainian)

10. O.M. Mulyava, M.M. Sheremeta, Relative growth of Dirichlet series// Mat. Stud. - 2018. - V.49, №2. P. $158-164$.

11. O.M. Mulyava, M.M. Sheremeta, Remarks to relative growth of entire Dirichlet series// Visnyk of Lviv Univ. Ser. mech.-math. $-2019 .-$ V.87. - P. 73-81.

12. Yu.M. Gal', M.M. Sheremeta, On the growth of analytic in half-plane fuctions given by Dirichlet series// Dopovidi AN URSR, ser. A. - 1978. - №12. - P. 1065-1067. (in Russian)

13. Yu.M. Gal', On the growth of analytic functions, represented by Dirichlet series absolutely convergent in half-plane. - Drogobych. - 1980. - 30 p. - Manuscr. Dep. in VININI 16.09.1980, № 4080-80Dep. (in Russian)

14. V.S. Boychuk, On the growth of Dirichlet series absolutely convergent in half-plane// Mat. sbornik. Kiev: Nauk. dumka. - 1976. - P. 238-240. (in Russian)

Kyiv National University of Food Technologies

Kyiv, Ukraine

oksana.m@bigmir.net

Received 12.07.2020

Revised 20.11.2020 